\title{
Space Time Block Code Classification for MIMO Signals Exploiting Cyclostationarity
}

\author{
Merve Turan \\ Department of Electronics and \\ Communications Engineering \\ Istanbul Technical University \\ Istanbul, Turkey \\ Email: turanmerv@itu.edu.tr
}

\author{
Mengüç Öner \\ Department of Electrical \\ and Electronics Engineering \\ Isik University \\ Istanbul, Turkey \\ Email: oner@isikun.edu.tr
}

\author{
Hakan Çrpan \\ Department of Electronics and \\ Communications Engineering \\ Istanbul Technical University \\ Istanbul, Turkey \\ Email: cirpanh@itu.edu.tr
}

\begin{abstract}
Blind and noncooperative identification of the transmission parameters of unknown communication signals has been employed both in military and civilian applications. Multiple-Input-Multiple-Output (MIMO) transmission systems emerging in the last decade pose new challenges to the signal identification systems, one of which is the identification of the Space-Time Block Code (STBC) used in the transmission. In this work, we present a novel STBC classification algorithm that exploits the joint wide sense cyclostationary characteristics of the coded transmit signals as discriminating features. Compared to existing algorithms, the proposed method can discriminate between a large number of different STBCs.
\end{abstract}

\section{INTRODUCTION}

The identification of the transmission parameters of unknown communication signals within the context of spectrum monitoring and surveillance, commonly referred to as signal identification, has been a popular and significant research area, and the methodologies developed for this purpose have been employed both in military and civilian applications. The main task of signal identification is the blind and non-cooperative extraction of the parameters specific to the transmission method used by the unknown transmitter, such as modulation type, carrier frequency, bandwidth, employed multiple access and frequency spreading methods, etc.

Because of the increasing diversity and complexity in the communication techniques employed in digital wireless communication systems, the existing methods used in signal identification need to be constantly extended and improved to include newly emerging wireless digital transmission techniques. The multiple input multiple output (MIMO) communication systems emerging in the last decade, which use multiple antennas for transmission, represent such an example, as they present new transmission parameters to be identified, which are not found in conventional single antenna transmission, such as the employed space-time block code (STBC) .

In this work, the problem of the STBC classification is investigated, which can be considered as a multiple hypothesis testing problem, with each hypothesis corresponding to a STBC from the set of possible STBC's $\Phi$, and the decision is made based on a finite number of observations of the received signal corrupted by fading and noise. Recently, a maximum likelihood approach has been proposed for the classification of the space-time block codes employed in a MIMO system [1], which, however, suffers from a high computational complexity, and requires a-priori information about the channel parameters, code timing (i.e. the beginning and the end of each transmitted code block), and the modulation type employed in the transmission, limiting its use in practice. In [2], a classification strategy is proposed based on the space-time correlations in the received signal induced by the coding operation, which, however, fails to acknowledge the cyclostationary nature of these correlations, that, if recognized and exploited properly, provides additional information for the classification task. In the binary hypothesis test proposed in [3] the cyclostationarity of the received MIMO signal induced by the space time block code is exploited for the first time for discriminating between space time coded and uncoded (i.e. spatially multiplexed) MIMO signals, however, discrimination between different codes is not considered. In [4], a STBC classification method is proposed, which provides robustness against transmission impairments by exploiting the cyclostationarity of the received signal vector, making use of the fact that STBCs of different block lengths exhibit cyclostationarity with different cyclic frequencies, however, discrimination between codes which exhibit cyclostationarity with the same cyclic frequency, (i.e.codes with the same block length) is not considered. In [5], the use of fourth order cyclic statistics of the received signal has been proposed for discriminating between Alamouti coded and spatially multiplexed MIMO signals, which requires only a single receive antenna.

In this work, we propose a novel STBC classification algorithm that, similar to [3] and [4], uses second order cyclostationary statistics as discriminating features. However, unlike those two algorithms, it estimates the discriminating features after blindly compensating the effects of the MIMO channel and recovering a noisy version of the original transmit signal, which leads to a finer discriminatory capability between different STBCs, and makes the algorithm capable of discriminating between the codes which have the same cycle frequencies, i.e. the STBCs with the same code block length. Thus, compared to the existing methods, the proposed algorithm is capable of discriminating between a larger number of codes. Furthermore, the algorithm does not require any apriori information on the number of transmit antennas, the modulation type of the transmit signals, the SNR or the channel matrix.

This work has been supported by the TUBITAK (The Scientific and Technological Research Council of Turkey) Grant Nr. 112E020 


\section{SySTEM MODEL}

In this work, we consider a MIMO system with $n_{t}$ transmit and $n_{r}$ receive antennas, where $n_{r} \geq n_{t}$. The received signal vector at time instant $k, \mathbf{r}[k]=\left[r_{1}[k], \ldots, r_{n_{r}}[k]\right]^{T}$ can be expressed as:

$$
\mathbf{r}[k]=\mathbf{H s}[k]+\mathbf{w}[k]
$$

where $s[k]=\left[s_{1}[k], \ldots, s_{n_{t}}[k]\right]^{T}$ is the transmit signal vector of length $n_{t}, \mathbf{w}[k]$ is is the circular complex additive white Gaussian noise vector with variance $\sigma_{w}^{2}, \mathbf{H}$ is the $n_{r} \times n_{t}$ channel matrix, whose elements $[\mathbf{H}]_{i, j}$ represent the channel cofficients between $i$ 'th receive and $j$ 'th transmit antenna, and are modeled as independent zero-mean circular complex Gaussian random variables with unit variance. In this work, the MIMO channel is assumed to be a frequency flat block fading channel over the observation interval.Without loss of generality, we assume unit power transmit signals.

In a MIMO system using a STBC, the transmit signal vector $\mathbf{s}[k]$ is generated by parsing the independent and identically distributed modulated information bearing symbols $x[k]$ into blocks of length $Q$, and mapping them to the transmit antennas according to a coding rule, which can be represented by an $n_{t} \times N_{\text {block }}$ code matrix, where $N_{\text {block }}$ is the duration of the code block. In the case of spatial multiplexing (SM) MIMO systems, however, the information symbols are mapped directly to the transmit antennas, which can be considered as a special case of STBC with $Q=n_{t}$ and $N_{\text {block }}=1$.

The task of the proposed STBC classification algorithm is to identify, from a set of different possible STBCs $\Phi$, the STBC that is being used in the received unknown MIMO signal. The code matrices of the STBCs considered in this work are listed in the Appendix. We consider codes designed for $n_{t}=2,3$ and 4 and block lengths $N_{\text {block }}=2,4$ and 8 . Furthermore, the case of SM is considered separately for each $n_{t}$.

\section{The STBC Classification Strategy}

In this work, we propose to exploit the joint wide-sense cyclostationary characteristics of the components of the transmit signal vector $\mathbf{s}[k]$, which is induced by the space-time block coding operation, as discriminating features for classification.

\section{A. Cyclostationarity:Preliminaries}

Two discrete time random processes $s_{1}[k]$ and $s_{2}[k]$ are called jointly wide-sense cyclostationary if either the time varying cross correlation function (TVCCF) $R_{s_{1} s_{2}}[k, \tau]=$ $E\left\{s_{1}[k] s_{2}^{*}[k-\tau]\right\}$ or the conjugate time varying cross correlation function (CTVCCF) $R_{s_{1} s_{2}^{*}}[k, \tau]=E\left\{s_{1}[k] s_{2}[k-\tau]\right\}$ (or both) exhibits a periodicity in the time index $k$. Clearly, such a periodicity allows the following Fourier series representation for the TVCCF and the CTVCCF functions:

$$
R_{s_{1} s_{2}}[k, \tau]=\sum_{\alpha=2 \pi l / K} R_{s_{1} s_{2}}^{\alpha}[\tau] e^{j \alpha k},
$$

and

$$
R_{s_{1} s_{2}^{*}}[k, \tau]=\sum_{\alpha=2 \pi l / L} R_{s_{1} s_{2}^{*}}^{\alpha}[\tau] e^{j \alpha k}
$$

where $K$ and $L$ are the fundamental periods of the respective crosscorrelation functions, and the sums are taken over the integer multiples of the fundamental cyclic frequencies $\alpha_{0}=2 \pi / K$ and $\alpha_{0}^{\prime}=2 \pi / L$ respectively. The Fourier series coefficients, $R_{s_{1} s_{2}}^{\alpha}[\tau]$ and $R_{s_{1} s_{2}^{*}}^{\alpha}[\tau]$, which depend on

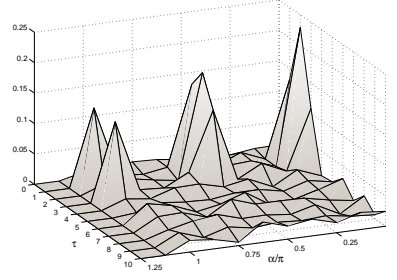

(a) $\left|R_{s_{1} s_{3}^{*}}^{\alpha}(\tau)\right|$ for $C_{1}$

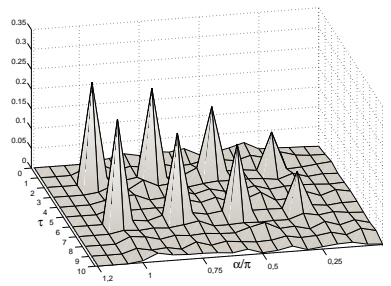

(c) $\left|R_{s_{1} s_{3}^{*}}^{\alpha}(\tau)\right|$ for $C_{2}$

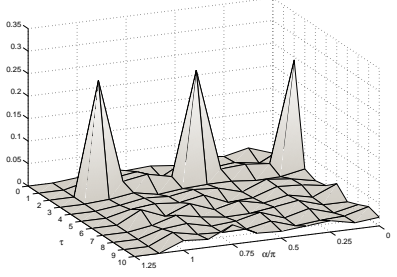

(b) $\left|R_{s_{1} s_{3}^{*}}^{\alpha}(\tau)\right|$ for $C_{3}$

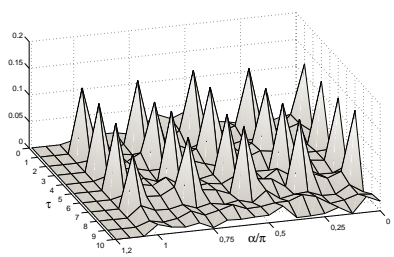

(d) $\left|R_{s_{1} s_{4}^{*}}^{\alpha}(\tau)\right|$ for $C_{4}$
Fig. 1. The CCCF functions between different components of the transmit signal for various STBCs.

the cycle frequency parameter $\alpha$ and the lag parameter $\tau$ are referred to as the cyclic crosscorrelation function (CCF) ad the conjugate cyclic crosscorrelation function (CCCF) respectively. Obviously, these functions are only nonzero for the values of $\alpha$ which are integer multiples of the fundamental cyclic frequency, and for processes, which do not exhibit joint cyclostationarity, $R_{s_{1} s_{2}}^{\alpha}[\tau]=R_{s_{1} s_{2}^{*}}^{\alpha}[\tau]=0 \forall \alpha, \tau$. Two random processes are said to exhibit joint conjugate widesense cyclostationarity at a specific pair $\left(\alpha=\alpha_{i}, \tau=\tau_{j}\right)$ if $R_{s_{1} s_{2}^{*}}^{\alpha_{i}}\left[\tau_{j}\right] \neq 0$. Similarly, two random processes are said to exhibit joint (nonconjugate) wide-sense cyclostationarity at a specific pair $\left(\alpha=\alpha_{i}, \tau=\tau_{j}\right)$ if $R_{s_{1} s_{2}}^{\alpha_{i}}\left[\tau_{j}\right] \neq 0$.

\section{B. The Classification System}

It can be shown that the space-time block coding operation performed by the MIMO transmitter induces joint wide-sense cyclostationarity between the components of the transmit signal vector $\mathbf{s}[k]$, with a fundamental cycle frequency $2 \pi / N_{\text {block }}$. Figures 1(a) to (d) display examples of the the CCCF functions for various signal component pairs for codes $C_{1}, C_{2}, C_{3}$ and $C_{4}$ (for the corresponding code matrices, see equations (8), (9), (10) and (11) in the Appendix). It can be shown that for each code, the CCCF and CCF functions present unique patterns on the $(\alpha, \tau)$ plane. These patterns are independent of the modulation type employed and only depend on the structure of the corresponding STBC, and therefore, can be exploited for discriminating between different codes. In this work, we propose using the presence or absence of joint wide sense (conjugate and non-conjugate) cyclostationarity between the components of the transmit signal vector $\mathbf{s}[k]$ at specific $(\alpha, \tau)$ pairs as features for classification.

It should be noted that at the receiver, the signal $\mathbf{s}[k]$ is not available, and although the components of the received signal $\mathbf{r}[k]$ also exhibit joint cyclostationarity with the same cyclic frequency as the transmit signal $\mathbf{s}[k]$, the code-specific patterns described above cannot be recovered from $\mathbf{r}[k]$ due to the fact that each receive antenna receives a linear mixture 


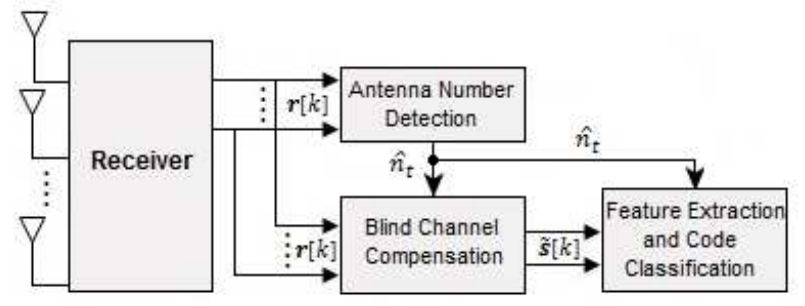

Fig. 2. The Block Diagram of the Proposed STBC Classfication Algorithm.

of the components of the transmit signal, which leads to a self-interference between the components of the transmit vector, making the discrimination between the codes with the same fundamental cycle frequencies impractical. Thus, the classification approach proposed in this work requires the blind compensation of the channel effects in order to recover a noisy version of the transmit signal, which in turn, is used for estimating the code-specific features. In this work, we propose to use blind source separation (BSS) techniques to recover the transmit signal from the noisy linear mixture $\mathbf{r}[k]$ prior to the classification. However, most of the BSS techniques found in the literature require a-priori knowledge about the number of sources, i.e., in our case, the number of the transmit antennas used in the transmission. Since this information is not available at the receiver due to the noncooperative nature of the scenarios considered in this work, the number of antennas needs to be detected prior to the channel compensation. The information on the number of antennas, besides being required for the blind channel compensation, can also be used as a feature for discriminating between different subsets of codes. Since each STBC is designed for a specific number of transmit antennas $n_{t}$, the knowledge of the number of antennas reduces the set of all possible codes $\Phi$, amongst which the unknown STBC is to be classifed, to only those designed for that specific $n_{t}$. Figure 2 displays the structure of the STBC classification algorithm proposed in this work. First, the number of transmit antennas $n_{t}$ is extracted from the received signal. This information is used for blindly compensating the channel effects and recovering a noisy version of the transmit signal, $\tilde{\mathbf{s}}[k]$, which in turn, along with the estimate of $n_{t}$, is used for the estimation of the codespecific features and classification.

\section{Detection of The Number of Transmit ANTENNAS}

Detecting the number of narrow band sources impinging on an array of sensors is a well investigated problem in the context of array signal processing and bearing estimation. The most common approach for this task found in existing literature is to use an information theoretic criterion such as the minimum description length (MDL) [6]. The information theoretic approach employed in MDL is a general approach for choosing a model that best fits the data from a family of possible models. The MDL criterion for the antenna number detection can be formulated as:

$$
\begin{aligned}
\hat{n}_{t} & =\arg \min _{k=1,2, \ldots}\left\{-\log \left(\Lambda_{\mathbf{R}}\left(\mathbf{r}[0], \ldots, \mathbf{r}[N-1] \mid \hat{\mathbf{H}}_{k}, \hat{\sigma}_{k}^{2}\right)\right.\right. \\
& \left.+0.5\left(2 k n_{r}+1\right) \log (N)\right\}
\end{aligned}
$$

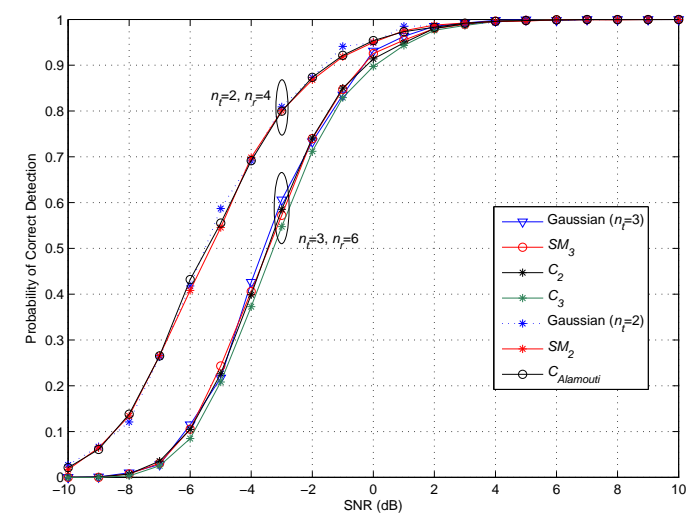

Fig. 3. Antenna number detection using GMDL for Gaussian and several non-Gaussian signals.

where $\Lambda_{\mathbf{R}}($.$) denotes the likelihood function of the received$ signal, and $\hat{\mathbf{H}}_{k}$ and $\hat{\sigma}_{k}^{2}$ are the maximum likelihood estimates of the channel matrix and the noise variance for the $k$ 'th hypothesis, respectively and $N$ is the length of the observation interval (See [6] and [7] for details on the general MDL problem).

Clearly, for the case considered in this work, where the transmit signals are space-time coded digitally modulated signals from a discrete alphabet, using the exact likelihood function of the received signal in the MDL criterion for antenna number detection requires a large amount of a-priori information such as the modulation type, the code block timing (i.e. the beginning and the end of each code block), and the employed STBC, which are unknown in our scenario (see, for example [8]). Furthermore, minimizing (4) over all possible transmit signals for this case would require a high computational power, especially for higher order modulations and a large $n_{t}$. These two issues limits the use of the exact MDL for this task in practice. However, for complex gaussian distributed source signals, the MDL criterion reduces to a simple closed form expression and depends only on the second order statistics of the received signal.

$$
\begin{gathered}
\hat{n}_{t}=\underset{k=1,2, \ldots, n_{r}-1}{\arg \min _{1}}-\log \left(\frac{\prod_{i=k+1}^{n_{r}} l_{i}^{\frac{1}{\left(n_{r}-k\right)}}}{\frac{1}{\left(n_{r}-k\right)} \sum_{i=k+1}^{n_{r}} l_{i}}\right)^{\left(n_{r}-k\right) N} \\
+\frac{1}{2} k\left(2 n_{r}-k\right) \log N
\end{gathered}
$$

where $l_{1}>l_{2}>\ldots>l_{n_{r}}$ represent the ordered eigenvalues of the estimated sample correlation matrix of the received signal, $\hat{\mathbf{R}}=\frac{1}{N} \sum_{k=0}^{N-1} \mathbf{r}[k] \mathbf{r}[k]^{\dagger}$ [6]. Clearly, this MDL detector, commonly referred to as the Gaussian MDL (GMDL) requires very little a-priori information and much less computational power.

In [7] it has been shown that the GMDL is not very sensitive to the actual distribution of the transmit signals, and can be used for source number detection even when the transmit signals are known to be non-Gaussian, if using the exact distribution of the transmit signals turns out to be prohibitive in practice. In Fig. 3, the use of GMDL for detecting the number of transmit antennas in a MIMO system with space time coding has been investigated via Monte Carlo 
simulations, where the detection performance of GMDL with gaussian transmit signals is compared to the GMDL with QPSK modulated MIMO signals in a block fading Rayleigh channel for $n_{t}=2$ and 3 , and an observation length of $N=1000$ symbols. For $n_{t}=2$, the codes $S M_{2}$ and $C_{\text {Alamouti }}$ are employed, and a receiver with $n_{r}=4$ is chosen, whereas for $n_{t}=3 S M_{3}$ and $C_{2}$ and $C_{3}$ has been used with $n_{r}=6$ (see Appendix for the corresponding code matrices). The simulation results show that both for $n_{t}=2$ and $n_{t}=3$, the loss in detection performance for nongaussian cases is very small compared to the gaussian case. Clearly, the use of the GMDL for the antenna number detection for MIMO communication signals leads to a good detection performance with reasonable computational complexity. Hence, we employ the GMDL detector in the proposed classification algorithm in the antenna number detection block.

\section{BLIND CHANNEL COMPENSATION}

In the MIMO context, the received signal can be considered as a linear mixture of the $n_{t}$ transmit signal components, each of which correspond to one of the transmit antennas, plus noise. The task of the blind channel compensation block is to separate this linear mixture into its components in order to recover a noisy version of the transmit signal, which can be then used for the extraction of code specific features. The framework of blind source separation (BSS) is a useful tool for this task. BSS is an umbrella term for computational methods employed for blindly separating linear mixtures of random processes into their individual components [9]. The various BSS algorithms found in the literature differ in the basic assumptions they make about the signal structure, the cost function they use for separating the signal components and the method used to solve the resulting optimization problem. In this work, we propose to use the joint approximate diagonalization of eigenmatrices (JADE) algorithm, which is a popular BSS method based on the maximization of a criterion function which employs the fourth order cumulant statistics of the received signal vector [10]. Although initially designed with the assumption of statistically independent signal components, it has been shown in [11] that the JADE algorithm exhibits a robustness to the time dependencies between the transmit signal components introduced in the signal vector by the space time block coding operation. Note that the JADE algorithm, like many BSS techniques, inherently contains phase and permutation ambiguities [9], i.e. the components of the recovered signal vector contain random phase rotations and their order is randomly interchanged, which should be taken into account by the feature extraction and classification blocks.

\section{FEATURE EXTRACTION AND ClassifiCATION}

The task of the feature extraction and classification block is to classify the STBC employed in the transmit signal by using the estimate of the number of transmit antennas $\hat{n}_{t}$ and the recovered transmit signal vector $\tilde{\mathbf{s}}[k]$, which contains phase and permutation ambiguities. The information on the number of transmit antennas $n_{t}$ is used at the classification subsystem as a feature to discriminate between the sets of codes designed for different $n_{t}$, reducing the set of possible STBCs considered in the classification to the set of codes designed for that particular number of transmit antennas. Thus, the task of feature extraction and classification can be tackled for each value of $\hat{n}_{t}$ separately. For each case, we

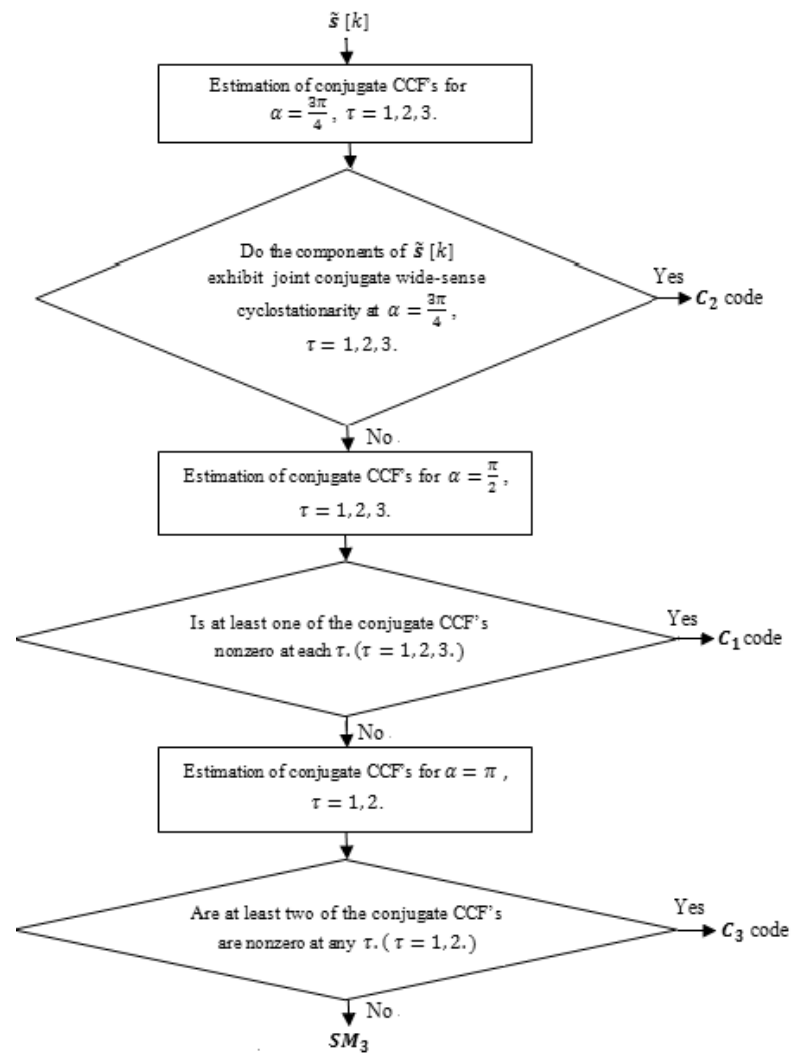

Fig. 4. The decision tree employed in classification for $\hat{n}_{t}=3$.

estimate the $\mathrm{CCCF}$ and/or $\mathrm{CCF}$ of the components of the recovered signal vector at specific $(\alpha, \tau)$ pairs, appropriately chosen to enable discrimination between different codes, and employ the presence or absence of cyclostationarity at those $(\alpha, \tau)$ pairs as discriminating features for classification. The presence of cyclostationarity at each $(\alpha, \tau)$ pair is detected by using the generalized likelihood ratio test (GLRT) proposed by Giannakis and Dandawate in [12], modified for detecting joint cyclostationarity. The insensivity of the GLRT to phase offsets ensures the robustness of the classification algorithm to the phase ambiguities discussed above.

A. Classification for $\hat{n}_{t}=2$

For $\hat{n}_{t}=2$, the classification is performed within the set $\Theta_{2}=\left\{S M_{2}, C_{\text {Alamout }}\right\}$, thus the classification problem reduces to a binary hypothesis test. As discussed in section III, the signal vector $\tilde{\mathbf{s}}[k]$ does not exhibit any joint wide sense cyclostationarity in the case of $S M_{2}$, whereas it is nonzero for $\alpha=\pi$ and $\tau=1$ for the Alamouti code. Thus for this case, it is sufficient to detect the absence or presence of cyclostationarity at $(\alpha=\pi, \tau=1)$ for the decision between the two hypotheses. Note that for $\hat{n}_{t}=2$, the permutation ambiguities in $\tilde{\mathbf{s}}[k]$ can be ignored.

\section{B. Classification for $\hat{n}_{t}=3$}

For $\hat{n}_{t}=3$, the classification is performed within the set $\Theta_{3}=\left\{S M_{3}, C_{1}, C_{2}, C_{3}\right\}$. It should be noted that for $n_{t}>2$, the permutation ambiguity in $\tilde{\mathbf{s}}[k]$ should be taken into account in the classification block, since a cross correlation pattern between two specific components of the transmit signal $\mathbf{s}[k]$ may appear between two different components of the recovered 
signal vector $\tilde{\mathbf{s}}[k]$. Thus, instead of looking for a specific cyclic cross correlation pattern between a specific antenna pair for discrimination, all three distinct CCCF functions are estimated for each distinct pair of the components of $\tilde{\mathbf{s}}[k]$, and common code-specific patterns in these CCCFs are exploited. For the set $\Theta_{3}=\left\{S M_{3}, C_{1}, C_{2}, C_{3}\right\}$, we choose the cyclic frequencies $\alpha=3 \pi / 4, \pi / 2$ and $\pi$ and $\tau=1,2$ and 3.The classification algorithm for this case is summarized in the decision tree given in Fig.4. The discrimination of $C_{2}$ is performed by realizing that $C_{2}$ is the only code in $\Theta_{2}$ which exhibits conjugate cyclostationarity at $\alpha=3 \pi / 4$. Since both $C_{1}$ and $C_{3}$ exhibit conjugate cyclostationarity at $\alpha=\pi / 2$, the discrimination between these two codes is performed by using the fact that two of the three CCCF functions of $C_{2}$ are nonzero for all three values of $\tau=1,2$ and 3 at $\alpha=\pi / 2$, whereas the CCCFs of $C_{3}$ at this cycle frequency are nonzero only for a single value of $\tau$. Finally the discrimination between $C_{3}$ and $S M_{3}$ can be easily performed by testing for conjugate cyclostationarity at $\alpha=\pi, \tau=1,2$, since spatial multiplexing signals do not exhibit cyclostationarity at all.

\section{Classification for $\hat{n}_{t}=4$}

For $\hat{n}_{t}=4$, the classification is performed within the set $\Theta_{4}=\left\{S M_{4}, C_{4}, C_{5}, C_{6}, C_{7}\right\}$. In this case, both the $\mathrm{CCF}$ an $\mathrm{CCCF}$ functions between the signal components (i.e. 6 of each) are required for classification. The code $C_{4}$ is easily discriminated, since it is the only code that exhibits conjugate cyclostationarity with $\alpha=3 \pi / 4$. Similarly, $C_{7}$ is discriminated by using the fact that it is the only code that exhibits a nonzero CCF at $\alpha=\pi$. The discrimination between $C_{5}$ and $C_{6}$ is performed by comparing the CCCF patterns at $\alpha=\pi$. The detailed decision tree for this case is omitted due to space restrictions.

\section{ClassificATION RESUltS}

In this section, the performance of the proposed algorithm is evaluated for a rayleigh block fading channel using simulations. We use the average probability of correct classification $P_{c c}$ as a performance measure, which is, assuming equiprobable hypotheses, given as

$$
P_{d}=\frac{1}{M} \sum_{j=1}^{M} P\left(H_{j} \mid H_{j}\right)
$$

Where $H_{j}$ represents the j'th hypothesis, each of which corresponds to one of the elements in the set of all possible codes $\Phi$ and $M$ is the cardinality of the set $\Phi$. In the simulations two different code sets are considered. The set $\Phi^{(1)}$ consisting of codes designed for $n_{t}=2$ and 3 , i.e. $\Phi^{(1)}=\Theta_{2} \cup \Theta_{3}=\left\{S M_{2}, C_{\text {Alamouti }}, S M_{3}, C_{1}, C_{2}, C_{3}\right\}$ (i.e $M=6$ ), and the set $\Phi^{(2)}$ containing STBCs designed for $n_{t}=2,3$, and 4, i.e. $\Phi^{(2)}=\Theta_{2} \cup \Theta_{3} \cup \Theta_{4}=$ $\left\{S M_{2}, C_{\text {Alamouti }}, S M_{3}, C_{1}, C_{2}, C_{3}, S M_{4}, C_{4}, C_{5}, C_{6}, C_{7}\right\}$ (i.e. $M=11$ ). In the simulations, 1000 Monte Carlo runs have been performed for each hypothesis, the observation length of the signal blocks used for classification is chosen as $N=1000,1500$ and 2000 and QPSK modulation is employed. Note that for the antenna number detection using GMDL, $n_{r}>n_{t}$ is required. Thus, in the simulations for the set $\Phi^{(1)}, n_{r}=4$ and 6 is chosen, whereas for the set $\Phi^{(2)}$, $n_{r}=6$ and 8 is considered. Furthermore, for the GLRT, the false alarm rate has been chosen as $P_{f a}=0.01$. The

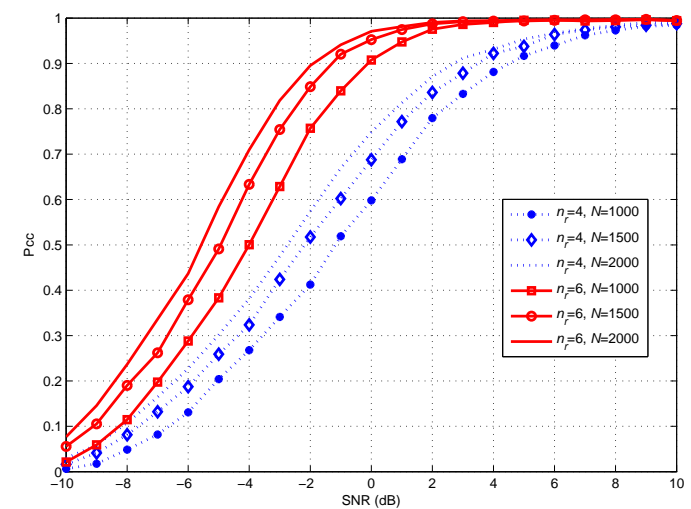

Fig. 5. The classification performance of the proposed algorithm for the set $\Phi^{(1)}(M=6)$.

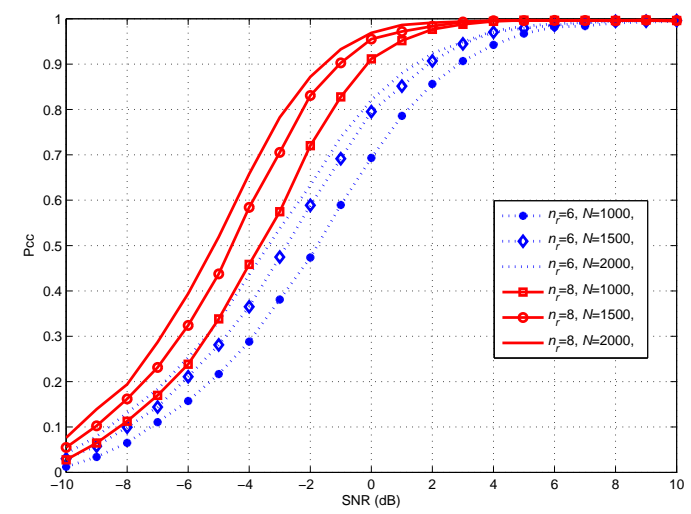

Fig. 6. The classification performance of the proposed algorithm for the set $\Phi^{(2)}(M=11)$.

classification performance of the proposed algorithm for the set $\Phi^{(1)}$ is displayed in Fig.5. Clearly, a good classification performance is achieved for relatively low SNR values, especially for $n_{r}=6$. Fig. 6 displays the classification results for the set $\Phi^{(2)}$, which, similarly, exhibit a good classification accuracy for both $n_{r}=6$ and 8 . Both figures show that the classification performance is highly dependent on the number of receive antennas, due to he fact that both the channel compensation and the antenna number detection blocks are sensitive to $n_{r}$. As expected, the classification performance increases in both cases, as the observation length $N$ increases.

\section{CONCLUSION}

A novel STBC classification algorithm is proposed that exploits the joint wide-sense cyclostationary behaviour induced in the transmit signal by the space time block coding operation. Since the extraction of the code specific cyclic correlation patterns requires the recovery of the transmit signal, blind channel compensation is performed prior to the feature extraction, which, in turn, requires the blind detection of the number of transmit antennas. This task is performed using the GMDL, an information theoretic criterion. Additionally, the estimate of the number of transmit antennas is used as a feature in the code classification for discriminating between codes 
designed for different numbers of transmit antennas. Compared to the existing methods in the literature, the proposed algorithm is capable of classifying a large number of different codes, and exhibits good classification performance for relatively low values of SNR.

\section{APPENDIX}

THE STBCS CONSIDERED FOR CLASSIFICATION

The classification algorithm proposed in this work is capable of discriminating between 11 different codes (note that spatial multiplexing for a given number of transmit antennas is also considered as a distinct code). The STBCs considered in this work are listed below, categorized by the number of transmit antennas they are used with.

\section{A. STBCs designed for $n_{t}=2$}

The set of space time block codes considered for $n_{t}=2$ is $\Theta_{2}=\left\{S M_{2}, C_{\text {Alamouti }}\right\}$, where $S M_{2}$ represents spatial multiplexing with two transmit antennas, and the code matrix of the Alamouti code designed for $n_{t}=2$ is given as [13]

$$
\mathbf{C}_{\text {Alamouti }}=\left[\begin{array}{cc}
x_{1} & -x_{2}^{*} \\
x_{2} & x_{1}^{*}
\end{array}\right]
$$

B. STBCs designed for $n_{t}=3$

The set of space time block codes considered for $n_{t}=3$ is $\Theta_{3}=\left\{S M_{3}, C_{1}, C_{2}, C_{3}\right\}$, where $S M_{3}$ represents spatial multiplexing with three transmit antennas, and the code matrices of $C_{1}$ [14], $C_{2}$ [14] and $C_{3}$ [15] are given as

$$
\begin{aligned}
\mathbf{C}_{1} & =\left[\begin{array}{rrrrrrr}
x_{1} & -x_{2}{ }^{*} & \frac{x_{3}^{*}}{\sqrt{2}} & & \frac{x_{3}^{*}}{\sqrt{2}} \\
x_{2} & x_{1}^{*} & \frac{x_{3}^{*}}{\sqrt{2}} & \frac{-x_{3}^{*}}{\sqrt{2}} \\
\frac{x_{3}}{\sqrt{2}} & \frac{x_{3}}{\sqrt{2}} & \frac{-x_{1}-x_{1}^{*}+x_{2}-x_{2}{ }^{*}}{2} & \frac{x_{2}+x_{2}^{*}+x_{1}-x_{1}{ }^{*}}{2}
\end{array}\right], \\
\mathbf{C}_{2} & =\left[\begin{array}{rrrrrrrr}
x_{1} & -x_{2} & -x_{3} & -x_{4} & x_{1}^{*} & -x_{2}^{*} & -x_{3}^{*} & -x_{4}^{*} \\
x_{2} & x_{1} & x_{4} & -x_{3} & x_{2}^{*} & x_{1}^{*} & x_{4}^{*} & x_{3}^{*} \\
x_{3} & -x_{4} & x_{1} & x_{2} & x_{3}^{*} & -x_{4}^{*} & x_{1}^{*} & x_{2}^{*}
\end{array}\right],
\end{aligned}
$$

and

$$
\mathbf{C}_{3}=\left[\begin{array}{crrr}
x_{1} & 0 & x_{2} & -x_{3} \\
0 & x_{1} & x_{3}^{*} & x_{2}^{*} \\
-x_{2}{ }^{*} & -x_{3} & x_{1}^{*} & 0
\end{array}\right]
$$

\section{STBCs designed for $n_{t}=4$}

The set of space time block codes considered for $n_{t}=4$ is $\Theta_{4}=\left\{S M_{4}, C_{4}, C_{5}, C_{6}, C_{7}\right\}$, where $S M_{4}$ represents spatial multiplexing with four transmit antennas, and the code matrices of the codes $C_{4}$ [14], $C_{5}$ [16], $C_{6}$ [17] and $C_{7}$ [18] are given as

$$
\mathbf{C}_{4}=\left[\begin{array}{rrrrrrrr}
x_{1} & -x_{2} & -x_{3} & -x_{4} & x_{1}^{*} & -x_{2}^{*} & -x_{3}^{*} & -x_{4}^{*} \\
x_{2} & x_{1} & x_{4} & -x_{3} & x_{2}^{*} & x_{1}^{*} & x_{4}^{*} & -x_{3}^{*} \\
x_{3} & -x_{4} & x_{1} & x_{2} & x_{3}^{*} & -x_{4}^{*} & x_{1}^{*} & x_{2}^{*} \\
x_{4} & x_{3} & -x_{2} & x_{1} & x_{4}^{*} & x_{3}^{*} & -x_{2}^{*} & x_{1}^{*}
\end{array}\right]
$$

$$
\begin{gathered}
\mathbf{C}_{5}=\left[\begin{array}{rrrr}
x_{1} & -x_{2}^{*} & x_{3} & -x_{4}^{*} \\
x_{2} & x_{1}^{*} & x_{4} & x_{3}^{*} \\
x_{3} & -x_{4}^{*} & x_{1} & -x_{2}^{*} \\
x_{4} & x_{3}^{*} & x_{2} & x_{1}^{*}
\end{array}\right], \\
\mathbf{C}_{6}=\left[\begin{array}{rrrr}
x_{1} & x_{2}^{*} & x_{3} & x_{4}^{*} \\
x_{2} & -x_{1}^{*} & -x_{4} & x_{3}^{*} \\
x_{3} & x_{4}^{*} & -x_{1} & -x_{2}^{*} \\
x_{4} & -x_{3}^{*} & x_{2} & -x_{1}^{*}
\end{array}\right],
\end{gathered}
$$

and

$$
\mathbf{C}_{7}=\left[\begin{array}{rrrr}
x_{1} & -x_{2}^{*} & -x_{3}^{*} & x_{4} \\
x_{2} & x_{1}^{*} & -x_{4}^{*} & -x_{3} \\
x_{3} & -x_{4}^{*} & x_{1}^{*} & -x_{2} \\
x_{4} & x_{3}^{*} & x_{2}^{*} & x_{1}
\end{array}\right] .
$$

\section{REFERENCES}

[1] V. Choqueuse, M. Marazin, L. Collin, K. Yao, and G. Burel, "Blind Recognition of Linear Space-Time Block Codes:A Likelihood-Based Approach,” IEEE Trans. Signal Process., vol. 58, no. 3, pp. 1290-1299, Mar. 2010.

[2] V. Choqueuse, K. Yao, L. Collin, and G. Burel, "Hierarchial Space Time Block Code Recognition Using Correlation Matrices," IEEE Trans. Wireless Commun., vol. 7, no. 9, pp. 3526-3534, Sep. 2008.

[3] M. Shi, Y. Bar-Ness, and W. Su, "STC and BLAST MIMO Modulation Recognition." in Proc. IEEE GLOBECOM Conf., Nov. 2007, pp. 3034-3039.

[4] M. Marey, O. A. Dobre, and R. Inkol, "Classification of Space-Time Block Codes Based on Second-Order Cyclostationarity with Transmission Impairments," IEEE Trans. Wireless Commun., vol. 11, no. 7, pp. 2574-2584, Jul. 2012.

[5] Y. A. Eldemerdash, M. Marey, O. A. Dobre, G. K. Karagiannidis, and R. Inkol, "Fourth-Order Statistics for Blind Classification of Spatial Multiplexing and Alamouti Space-Time Block Code Signals," IEEE Trans. Commun., vol. 61, no. 6, pp. 2420-2431, Jun. 2013.

[6] M. Wax and T. Kailath, "Detection of Signals by Information Theoretic Criteria," IEEE Transactions on Acoustics, Speech and Signal Processing, vol. 33, no. 2, pp. 387-392, Apr. 1985.

[7] E. Fishler, M. Grosmann, and H. Messer, "Detection of Signals by Information Theoretic Criteria: General Asymptotic Performance Analysis," IEEE Trans. Signal Process., vol. 50, no. 5, pp. 1027-1036, May. 2002.

[8] E. Fishler, M. Grosman, and H. Messer, "Determining the Number of Discrete Alphabet Sources from Sensor Data," EURASIP Journal on Applied Signal Processing, vol. 2005, pp. 4-12, Jan. 2005.

[9] P. Comon and C. Jutten, Eds., Handbook of Blind Source Separation: Independent Component Analysis and Applications. Elsevier, 2010.

[10] J. F. Cardoso and A. Souloumiac, "Blind Beamforming for NonGaussian Signals," IEE Proceedings F Radar and Signal Processing, vol. 140, no. 6, pp. 362-370, Dec. 1993.

[11] G. Qian and L. Li, "On the blind channel identifiability of multipleinput multiple-output spacetime block code systems using joint approximate diagonalization of eigenmatrices," Wireless Communications and Mobile Computing, Oct. 2013.

[12] A. V. Dandawate and G. B. Giannakis, "Statistical Tests for Presence of Cyclostationarity," IEEE Trans. Signal Process., vol. 42, no. 9, pp. 355-2369, Sep. 1994.

[13] S. Alamouti, "A Simple Transmit Diversity Technique for Wireless Communications," IEEE Journal on Selected Areas in Communications, vol. 16, no. 8, pp. 1451-1458, Oct. 1998.

[14] V. Tarokh, H. Jafarkhani, and A. R. Calderbank, "Space-Time Block Codes from Orthogonal Designs," IEEE Trans. Inform. Theory, vol. 45, no. 5, pp. 1456-1467, Jul. 1999.

[15] E. G. Larsson and P. Stoica, Space-Time Block Coding for Wireless Communications. Cambridge University Press, 2003., p.104.

[16] O. Tirkkonen, A. Boariu, and A. Hottinen, "Minimal Non-Orthogonality Rate 1 Space-Time Block Code for 3+ Tx Antennas," vol. 2. IEEE Sixth International Symposium on Spread Spectrum Techniques and Applications, Sep. 2000, pp. 429-432.

[17] C. B. Papadias and G. J. Foschini, "Capacity-Approaching Space-Time Codes for Systems Employing Four Transmitter Antennas," IEEE Trans. Inform. Theory, vol. 49, no. 3, pp. 726-732, Mar. 2003.

[18] H. Jafarkhani, "A Quasi-Orthogonal SpaceTime Block Code," IEEE Trans. Commun., vol. 49, no. 1, pp. 1-4, Jan 2001. 